\title{
Single-Plasmon Thermo-Optical Switching in Graphene
}

\author{
Joel D. Cox ${ }^{*,+, \ldots, \Phi}$ and F. Javier García de Abajo*,+,ß \\ $\dagger I C F O$-Institut de Ciencies Fotoniques, The Barcelona Institute of Science and Technology, \\ 08860 Castelldefels (Barcelona), Spain \\ $\ddagger$ Center for Nano Optics, University of Southern Denmark, Campusvej 55, DK-5230 \\ Odense $M$, Denmark \\ IDanish Institute for Advanced Study, University of Southern Denmark, Campusvej 55, \\ DK-5230 Odense M, Denmark \\ §ICREA-Institució Catalana de Recerca i Estudis Avançats, Passeig Lluís Companys 23, \\ 08010 Barcelona, Spain \\ E-mail: cox@mci.sdu.dk; javier.garciadeabajo@nanophotonics.es
}

\begin{abstract}
While plasmons in noble metal nanostructures enable strong light-matter interactions on nanometer length scales, the overabundance of free electrons in these systems inhibits their tunability by weak external stimuli. Countering this limitation, the linear electronic dispersion in graphene endows the two-dimensional material with both an enhanced sensitivity to doping electron density, enabling active tunability of its highlyconfined plasmon resonances, and a very low electronic heat capacity that renders its thermo-optical response extraordinarily large. Here we show that these properties combined enables a substantial optical modulation in graphene nanostructures from the energy associated with just one of their supported plasmons. We base our analysis on realistic, complimentary classical and quantum-mechanical simulations, which
\end{abstract}


reveal that the energy of a single plasmon, absorbed in a small, moderately-doped graphene nanoisland, can sufficiently modify its electronic temperature and chemical potential to produce unity-order modulation of the optical response within subpicosecond timescales, effectively shifting or damping the original plasmon absorption peak and thereby blockading subsequent excitation of a second plasmon. The proposed thermo-optical single-plasmon blockade consists in a viable ultra-low power all-optical switching mechanism for doped graphene nanoislands, while their combination with quantum emitters could yield applications in biological sensing and quantum nanooptics.

Keywords: all-optical switching, graphene plasmonics, optical modulation, thermo-optical response, single-photon devices, quantum optics

The ability of plasmons -the collective oscillations of conduction electrons- to intensify the electromagnetic field of light has stimulated fertile research efforts in nonlinear plasmonics to control light by light on the nanoscale. ${ }^{1}$ In this context, engineered plasmonic near field enhancement from noble metal nanostructures has been enormously successful in bolstering the weak susceptibilities associated with coherent nonlinear processes while reducing the intensity threshold for optical switching. ${ }^{2}$ However, despite the achievement of impressive levels of plasmonic enhancement, the realization of strong nonlinear optical interactions at ultra-low powers, ultimately down to the few- or single-photon regime, remains elusive. ${ }^{3-5}$

The enhanced light absorption associated with the resonant excitation of plasmons in noble metals stimulates the generation of energetic (hot) electrons and holes, which thermalize via electron-electron scattering on timescales of 10s of femtoseconds, reaching a local equilibrium state with an elevated electron temperature, followed by cooling at a much slower rate $(\sim 1 \mathrm{ps})$ by coupling to lattice vibrations. ${ }^{6-10}$ While rapid thermalization inhibits photochemical $^{11}$ and photovoltaic ${ }^{12}$ functionalities based on the separation and extraction of energetic electrons and holes, the transient plasmonic response associated with the local thermallized equilibrium electron distribution provides a potentially useful source of ultrafast 
thermal nonlinearity. ${ }^{13-15}$

In this context, electrically doped graphene -the atomically-thin carbon layer- appears as an excellent infrared plasmonic material ${ }^{16,17,17-23}$ in which a comparatively small number of electrons rule its optical and thermal properties in the ultrafast regime, thus displaying extraordinary thermo-optical properties that hold strong potential for all-optical modulation. ${ }^{21,24-28}$ Following excitation by an ultrafast optical pump, graphene plasmons decay on typically longer timescales than their noble metal counterparts into energetic electrons and holes, ${ }^{23,29}$ which subsequently thermalize on ultrafast timescales. ${ }^{25,26}$ Owing to its linear electronic dispersion relation, which endows graphene with a low electronic heat capacity, the large amount of optical energy absorbed through resonant excitation of plasmons can dramatically elevate the temperature of conduction electrons in the $2 \mathrm{D}$ material. ${ }^{21}$ The thermalized electronic distribution can be optically probed while electrons cool by gradually transferring their energy to phonons, essentially rendering the optical response nonlinear through its dependence on the primary excitation pump intensity. ${ }^{30,31}$

In this Letter we propose that the combined large thermo-optical response and very low electronic heat capacity originating from the unique conical dispersion of graphene can enable the modulation of the optical response upon excitation of a single plasmon. In particular, we reveal scenarios in which the energy of a single plasmon quantum $\left(\hbar \omega_{\mathrm{p}}\right.$, where $\omega_{\mathrm{p}}$ is the plasmon frequency), distributed after it inelastically decays among a finite number of electrons, can modify the local equilibrium temperature and chemical potential of a graphene nanoisland enough to produce substantial changes in its supported plasmon resonances. Remarkably, in small graphene nanoislands doped with only a few electrons, we predict unity-order modulation of the optical response through single-plasmon absorption on ultrafast timescales, extending over picosecond timescale, as determined by the release of electronic energy to lattice vibrations. This phenomenon consists in an incoherent plasmon blockade, whereby the decay of a single excited plasmon suppresses the probability of exciting additional plasmons. 
In what follows we outline the theoretical treatments used to describe the thermo-optical response associated with single-plasmon absorption in graphene nanoislands. For structures with dimensions $\gtrsim 10 \mathrm{~nm}$, we adopt a semi-analytical approach rooted in the quasistatic limit of classical electromagnetism, employing a local optical conductivity to describe the graphene response. ${ }^{32}$ In smaller graphene structures, we implement an atomistic description of the optical response, accounting for quantum and finite-size effects that become important in this regime. ${ }^{33}$ In either case, we estimate the change in optical response associated with single-plasmon absorption by a doped graphene nanostructure using the following procedure:

1. We identify the plasmon resonance frequency $\omega_{\mathrm{p}}$ from the simulated linear absorption spectum of a graphene nanoisland at zero temperature and doped to a Fermi energy $E_{\mathrm{F}}$.

2. The equilibrium chemical potential $\mu$ and electronic temperature $T$ of the nanoisland when it has absorbed the single-plasmon energy $\hbar \omega_{\mathrm{p}}$ are computed self-consistently, ensuring conservation of energy and electron population.

3. With the obtained values of $\mu$ and $T$, we re-calculate the graphene nanoisland absorption spectrum and observe the change in optical response relative to zero temperature.

This approach estimates the maximum possible change in optical response associated with the absorption of a single plasmon by assuming that it only decays to electron-hole pairs that rapidly thermalize via electron-electron scattering without losing energy.

Classical Treatment of Single-Plasmon Absorption in Graphene. Low-energy electrons in graphene follow a conical dispersion relation $E\left(\mathbf{k}_{\|}\right)=\hbar v_{\mathrm{F}}\left|\mathbf{k}_{\|}\right|$, where $v_{\mathrm{F}} \approx$ $10^{6} \mathrm{~m} / \mathrm{s}$ is the Fermi velocity and $\mathbf{k}_{\|}$the in-plane electron wave vector. At zero temperature, the optical conductivity at frequency $\omega$ is obtained in the local response limit (i.e., for vanishing in-plane optical momentum) of the random-phase approximation (RPA) as ${ }^{34,35}$

$$
\sigma_{\omega}^{(1)}=\frac{e^{2}}{\hbar}\left[\frac{\mathrm{i} E_{\mathrm{F}} /(\pi \hbar)}{\omega+\mathrm{i} \tau^{-1}}+\frac{1}{4} \Theta\left(\hbar \omega-2 E_{\mathrm{F}}\right)+\frac{\mathrm{i}}{4 \pi} \log \left(\frac{\omega+\mathrm{i} \tau^{-1}-2 E_{\mathrm{F}} / \hbar}{\omega+\mathrm{i} \tau^{-1}+2 E_{\mathrm{F}} / \hbar}\right)\right],
$$


where the Fermi energy $E_{\mathrm{F}}$ is related to the graphene doping charge-carrier density $n$ according to $E_{\mathrm{F}}=\hbar v_{\mathrm{F}} \sqrt{\pi n}$ and $\tau$ is a phenomenological inelastic scattering rate, for which we choose a conservative value $\left(\hbar \tau^{-1}=50 \mathrm{meV}\right)$ throughout this study. The first terms in eq 1 describe the optical response arising from intraband electronic transitions, whereas the remaining two terms correspond to interband transitions.

We consider a graphene nanostructure interacting with linearly-polarized monochromatic light of in-plane external electric field $E^{\mathrm{ext}} \mathrm{e}^{-\mathrm{i} \omega t} \hat{\mathbf{x}}+$ c.c., taken along a symmetry direction $\hat{\mathbf{x}}$. If the structure has characteristic size $D$ (e.g., the diameter of a disk or the side length of a square) and is doped to a Fermi energy $E_{\mathrm{F}}$, a localized plasmon mode with resonance frequency $\omega_{\mathrm{p}}$ is well-described when $\hbar \omega_{\mathrm{p}} \lesssim E_{\mathrm{F}}$ and $D \gtrsim 10 \mathrm{~nm}$. In this regime, assuming $D$ to be small compared with the light wavelength, an expansion of the electrostatic potential in orthonormal eigenmodes yields real eigenvalues $\eta_{j}$ and their associated plasmon wave functions (PWFs) $\rho_{j}(\mathbf{R} / D)$ corresponding to modes labeled by $j$ for a specific $2 \mathrm{D}$ morphology. ${ }^{21,32}$ In terms of PWFs, the linear polarizability can be analytically expressed $\operatorname{as}^{32}$

$$
\alpha_{\omega}^{(1)}=\sum_{j} \frac{\bar{\epsilon}_{\omega} \xi_{j}^{2} D^{3}}{1 / \eta_{\omega}^{(1)}-1 / \eta_{j}},
$$

where $\xi_{j}=\int d^{2} \mathbf{R} x \rho_{j}(\mathbf{R} / D)$ quantifies the coupling of mode $j$ with the external field, $\eta_{\omega}^{(1)}=$ $\mathrm{i} \sigma_{\omega}^{(1)} / \epsilon_{\omega} \omega D$ contains the dependence on the local, isotropic optical conductivity $\sigma_{\omega}^{(1)}$, and $\bar{\epsilon}_{\omega}$ is the background permittivity. This expression is also valid for graphene supported on a substrate with $\bar{\epsilon}_{\omega}$ set the average permittivity of the media on either side of the interface. ${ }^{21}$ With the tabulated parameters $\eta_{j}$ and $\xi_{j}$ reported in Ref. ${ }^{32}$ for specific $2 \mathrm{D}$ geometries and the conductivity entering $\eta^{(1)}$, the linear polarizability can be computed from eq 2 directly. The situation simplifies further if $\omega_{\mathrm{p}} \lesssim E_{\mathrm{F}}$ and we assume zero temperature, so that the effect of interband optical transitions can be reasonably neglected and we retain only the leading Drude-like term in the conductivity of eq 1; identifying plasmons in the poles of eq 2 , we 
obtain the resonance energy associated with the $j^{\text {th }}$ eigenmode as $\hbar \omega_{j}=e \sqrt{E_{\mathrm{F}} /\left(-\eta_{j} \bar{\epsilon}_{\omega} \pi D\right)}$.

We now consider a scenario in which a graphene layer doped to a Fermi energy $E_{\mathrm{F}}$ absorbs some thermal energy $Q$ that elevates the electronic temperature $T$ and conserves the doping charge density ${ }^{27}$

$$
n=\frac{g_{v} g_{s}}{2 \pi\left(\hbar v_{\mathrm{F}}\right)^{2}} \int_{0}^{\infty} d E E[f(E, \mu, T)+f(-E, \mu, T)-1]
$$

where $g_{v}=g_{s}=2$ are the valley and spin degeneracies and $f(E, \mu, T)=\left[\mathrm{e}^{(E-\mu) / k_{\mathrm{B}} T}+1\right]^{-1}$ is the Fermi-Dirac distribution for a chemical potential $\mu$. Equation 3 is obtained for electron doping, with the term $f$ in the square brackets accounting for the electron distribution in the partially-filled upper Dirac cone and $1-f$ describing the holes in the lower cone. Following a previously established procedure, ${ }^{27}$ we can recast eq 3 into an expression that implicitly relates $E_{\mathrm{F}}, T$, and $\mu$ as

$$
\begin{aligned}
\left(\frac{E_{\mathrm{F}}}{k_{\mathrm{B}} T}\right)^{2} & =2 \int_{0}^{\infty} d x x\left[\frac{1}{\mathrm{e}^{x-\mu / k_{\mathrm{B}} T}+1}-\frac{1}{\mathrm{e}^{x+\mu / k_{\mathrm{B}} T}+1}\right] \\
& =2\left[\operatorname{Li}_{2}\left(-\mathrm{e}^{-\mu / k_{\mathrm{B}} T}\right)-\operatorname{Li}_{2}\left(-\mathrm{e}^{\mu / k_{\mathrm{B}} T}\right)\right]
\end{aligned}
$$

where the second line is expressed in terms of the polylogarithm function $\mathrm{Li}_{2}$. Using the above result along with the graphene heat capacity ${ }^{27}$

$$
\frac{Q}{A}=\beta \frac{\left(k_{\mathrm{B}} T\right)^{3}}{\left(\hbar v_{\mathrm{F}}\right)^{2}},
$$

where

$$
\begin{aligned}
\beta & =\frac{2}{\pi}\left[\int_{0}^{\infty} d x x^{2}\left(\frac{1}{\mathrm{e}^{x+\mu / k_{\mathrm{B}} T}+1}+\frac{1}{\mathrm{e}^{x-\mu / k_{\mathrm{B}} T}+1}\right)-\frac{1}{3}\left(\frac{E_{\mathrm{F}}}{k_{\mathrm{B}} T}\right)^{3}\right] \\
& =\frac{2}{\pi}\left\{-2\left[\operatorname{Li}_{3}\left(-\mathrm{e}^{-\mu / k_{\mathrm{B}} T}\right)-\mathrm{Li}_{3}\left(-\mathrm{e}^{\mu / k_{\mathrm{B}} T}\right)\right]-E_{\mathrm{F}}{ }^{3} / 3\left(k_{\mathrm{B}} T\right)^{3}\right\},
\end{aligned}
$$

we obtain values of $\mu$ and $T$ conserving the net electronic population specified by the Fermi 
energy $E_{\mathrm{F}}$ when the electrons in a graphene layer with area $A$ absorb an energy $Q$.

For finite electron temperatures we use the local-RPA graphene conductivity ${ }^{27,36}$

$$
\sigma_{\omega}^{(1)}=\frac{e^{2}}{\pi \hbar^{2}} \frac{\mathrm{i}}{\omega+\mathrm{i} \tau^{-1}}\left\{\mu^{\mathrm{D}}-\int_{-\infty}^{\infty} d E \frac{E /|E|}{1-4 E^{2} /\left[\hbar^{2}\left(\omega+\mathrm{i} \tau^{-1}\right)^{2}\right]} f(E, \mu, T)\right\},
$$

where

$$
\mu^{\mathrm{D}}=\mu+2 k_{\mathrm{B}} T \log \left(1+\mathrm{e}^{-\mu / k_{\mathrm{B}} T}\right)
$$

is a Drude-weight energy. This expression reduces to eq 1 in the limit of vanishing temperature (i.e., $T=0, \mu=E_{\mathrm{F}}$ ).

Quantum-Mechanical Description. We adopt an atomistic tight-binding (TB) description of electron states in the RPA to simulate the optical response of small graphene nanoislands containing a finite number of carbon atoms $N{ }^{33}$ In this so-called TB-RPA approach, the TB single-electron states $|j\rangle$ with energies $E_{j}$ are expanded in the basis set of carbon $2 \mathrm{p}$ orbitals $|l\rangle$ located at sites $\mathbf{R}_{l}$ according to $|j\rangle=\sum_{l} a_{j l}|l\rangle$, with coefficients $a_{j l}$ weighing the amplitude of orbital $|l\rangle$ in state $|j\rangle$, and used to compute the first-order induced charge density

$$
\rho_{l}^{\text {ind }}=\sum_{l^{\prime}} \chi_{l l^{\prime}}^{(0)} \phi_{l^{\prime}}
$$

where

$$
\chi_{l l^{\prime}}^{(0)}=\frac{2 e^{2}}{\hbar} \sum_{j j^{\prime}}\left(f_{j^{\prime}}-f_{j}\right) \frac{a_{j l} a_{j^{\prime} l}^{*} a_{j l^{\prime}}^{*} a_{j^{\prime} l^{\prime}}}{\omega+\mathrm{i} \tau^{-1}-\left(E_{j}-E_{j^{\prime}}\right) / \hbar}
$$

is the non-interacting RPA susceptibility (the factor of 2 arises from spin degeneracy), involving the state occupation factors $f_{j}$, and

$$
\phi_{l}=-\mathbf{R}_{l} \cdot \mathbf{E}^{\mathrm{ext}}+\sum_{l^{\prime}} v_{l l^{\prime}} \rho_{l}^{\mathrm{ind}}
$$



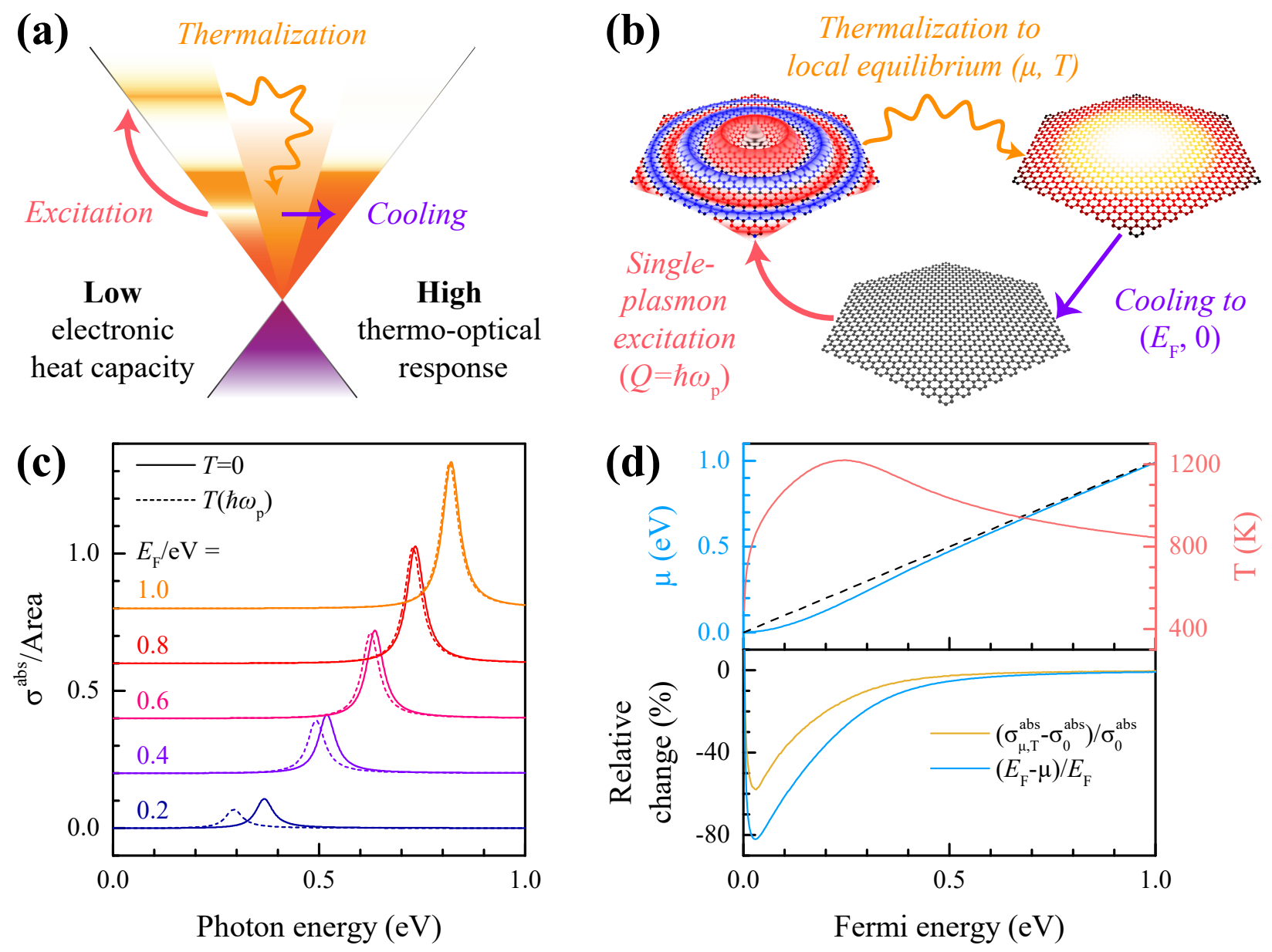

Figure 1: Single-plasmon thermo-optical switching. (a) Because of its unique conical dispersion relation, few hot carriers are needed to push a high electron temperature in graphene (low heat electronic capacity), also resulting in a large change of effective Drudeweight (large thermo-optical response). (b) Schematic illustration of an unperturbed, doped graphene nanohexagon at zero temperature (left image): a single plasmon quantum of energy $\hbar \omega_{\mathrm{p}}$ is excited (upper image) and absorbed, leading to a local thermal equilibrium state at chemical potential $\mu$ and electronic temperature $T$ after a few 10 s of fs (lower image), slowly returning to the original equilibrium state after transferring the absorbed energy to the carbon lattice $(\sim 1 \mathrm{ps})$. (c) Absorption cross section $\sigma^{\text {abs }}$ predicted in classical electrodynamic simulations (adopting the Drude conductivity with a phenomenological relaxation rate $\hbar \tau^{-1}=50 \mathrm{meV}$ ) for a nanohexagon of diameter $D=10 \mathrm{~nm}$ at Fermi energies indicated by the color-coordinates curves; in each case, we plot the response at zero temperature (solid curves), from which we identify the plasmon resonance frequency $\omega_{\mathrm{p}}$ from maxima in $\sigma^{\text {abs }}$, along with the response of the thermalized system for an excess energy $\hbar \omega_{\mathrm{p}}$ (dashed curves). (d) We present $\mu$ and $T$ (upper panel with color-coordinated axes and dashed curve indicating $\mu=E_{\mathrm{F}}$ ) along with the relative change in absorption and chemical potential (lower panel) upon absorption of the zero-temperature nanohexagon plasmon energy $\hbar \omega_{\mathrm{p}}$ as a function of $E_{\mathrm{F}}$. 
is the self-consistent electrostatic potential, with the first and second terms accounting for external and induced contributions, respectively, and $v_{l l^{\prime}}$ denoting the Coulomb interaction between electrons in $2 \mathrm{p}$ carbon orbitals located at $\mathbf{R}_{l}$ and $\mathbf{R}_{l^{\prime}}$. Numerical solution of eq 5 yields the induced charge, from which we characterize the optical response through the induced dipole moment $\mathbf{p}^{\text {ind }}=\sum_{l} \rho_{l}^{\text {ind }} \mathbf{R}_{l}$. We remark that the TB-RPA description of the optical response in small $(D \lesssim 10 \mathrm{~nm})$ graphene nanoislands is found to be in excellent qualitative agreement with the more rigorous time-dependent density functional theory. ${ }^{37}$

The occupation factors $f_{j}$ in eq 6 effectively weigh the single-electron transitions between states $|j\rangle$ and $\left|j^{\prime}\right\rangle$. At zero temperature $\left(f_{j}=f_{j}^{0}\right)$, states are populated by setting $f_{j}^{0}=1$ for $E_{j}<E_{\mathrm{F}}$ and $f_{j}^{0}=0$ for $E_{j}>E_{\mathrm{F}}$; additionally, if the Fermi level is degenerate, $f_{j}^{0}$ is set to a uniform fraction for states with $E_{j}=E_{\mathrm{F}}$ such that the total number of electrons $N_{\mathrm{e}}$ is preserved. When a heat energy $Q$ is added to the system, we assume thermal equilibrium $\left(f_{j}=f\left(E_{j}, \mu, T\right)\right)$ and impose conservation of energy,

$$
\sum_{j}\left(f_{j}-f_{j}^{0}\right) E_{j}=0
$$

and electronic population,

$$
N_{\mathrm{e}}=2 \sum_{j} f_{j}=\sum_{j} f_{j}^{0}
$$

Upon numerical solution of these eqs 7 and 8, we obtain $Q$-dependent values for $\mu$ and $T$.

In our study of single-plasmon thermo-optical switching we consider small graphene nanoislands with hexagonal geometries that can host exclusively armchair-type edge-terminations, thus avoiding detrimental effects to the strength and tunability of plasmon resonances arising from zero-energy states introduced in the electronic spectrum by zigzag edges. ${ }^{38,39} \mathrm{We}$ consider in Figure 1 graphene hexagons of diameter $D=10 \mathrm{~nm}$ undergoing the process illustrated schematically in Figure 1a,b: a nanohexagon at zero temperature doped to some Fermi energy $E_{\mathrm{F}}$ is resonantly excited and a single plasmon quantum with energy $\hbar \omega_{\mathrm{p}}$ decays 

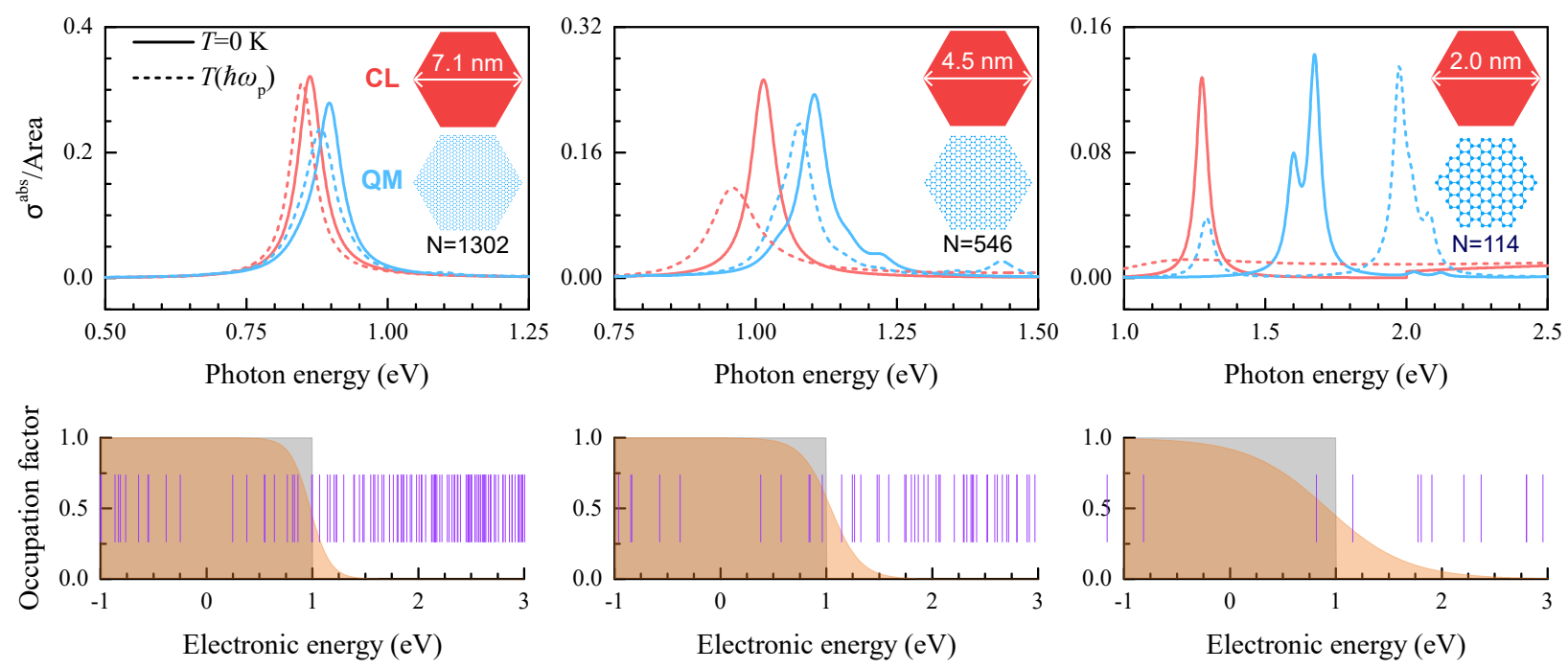

Figure 2: Single-plasmon absorption in classical and quantum-mechanical pictures. We plot the absorption spectra predicted in classical (CL, red curves) and quantummechanical (QM, blue curves) descriptions of graphene nanohexagons (upper row) with lateral sizes of $7.1 \mathrm{~nm}$ (left panel), $4.5 \mathrm{~nm}$ (center panel), and $2.0 \mathrm{~nm}$ (right panel), before (solid curves) and after (dashed curves) single plasmon absorption, indicating the number of carbon atoms $N$ of each exclusively armchair-edged island in the QM picture. For the QM simulations, we plot in the lower row the discrete spectrum of electronic states (vertical purple lines) and the electronic occupation factor before (gray filled curve) and after (orange filled curve) single-plasmon absorption for the hexagons considered in the upper row.

into electron-hole pairs that thermalize to yield a local equilibrium distribution with temperature $T$ and chemical potential $\mu$. We characterize the graphene nanoisland optical response from the absorption cross-section $\sigma^{\text {abs }}=(4 \pi \omega / c) \operatorname{Im}\left\{\alpha_{\omega}^{(1)}\right\}$, obtaining the linear polarizability $\alpha_{\omega}^{(1)}$ analytically from eq 2 by truncating the sum after the dominant $j=1$ dipolar plasmon mode and adopting the parameters $\eta_{1}=-0.0681$ and $\xi_{1}=0.7702$ associated with the hexagonal geometry. ${ }^{32}$ Throughout this work we assume a highly-conservative phenomenological relaxation rate $\hbar \tau^{-1}=50 \mathrm{meV}$ for graphene plasmons.

In Figure 1c we plot the graphene nanohexagon zero-temperature absorption spectra (solid curves) computed in the classical Drude description (i.e., retaining only the first term in eq 4) at various Fermi energies, yielding the single-plasmon energies $\hbar \omega_{\mathrm{p}}$, along with the corresponding spectra obtained for the thermalized electronic distribution resulting from single-plasmon absorption (dashed curves). Although the energy associated with a single 
plasmon $\hbar \omega_{\mathrm{p}}$ scales precisely with $\sqrt{E_{\mathrm{F}}}$ in the Drude conductivity considered here, more significant spectral shifts arise at lower Fermi energies, when the plasmon energy is distributed among fewer electrons. In Figure 1d we study the chemical potential $\mu$ and electronic temperature $T$ (upper panel) along with the relative changes in $\mu$ and light absorption at the plasmon energy (lower panel) attained through single-plasmon absorption as functions of the Fermi energy. At very low doping levels, negligible plasmon energies lead to only minor changes in $\mu$ and $T$, while the situation quickly changes as the Fermi energy increases to $E_{\mathrm{F}} \sim 0.2 \mathrm{eV}$, where a maximum electron temperature of $\sim 1300 \mathrm{~K}$ is achieved. Significant changes in absorption and chemical potential also take place towards lower Fermi energies but vanish for zero doping, when the plasmon energy also vanishes, and we note that in the Drude conductivity implemented here, the relative change in resonance frequency scales strictly proportionally to the change in chemical potential.

The results of Figure 1 indicate that at higher doping levels the benefit of a increased plasmon energy is overcome by the division of this energy among additional electrons. However, as plasmon resonances in doped graphene nanostructures scale according to $\propto \sqrt{E_{\mathrm{F}} / D}$, the energy per doping electron obtained from single-plasmon decay should increase in smaller islands. The electronic structure of graphene islands with dimensions $\lesssim 10 \mathrm{~nm}$ is strongly dependent on quantum finite-size and edge-termination effects, warranting their description in an quantum-mechanical (QM) framework. ${ }^{33}$ In Figure 2 we explore the classical-to-quantum transition in single-plasmon thermo-optical switching by comparing nanohexagon absorption spectra (upper row), calculated at a fixed Fermi energy $E_{\mathrm{F}}=1.0 \mathrm{eV}$, before (solid curves) and after (dashed curves) single-plasmon absorption in the classical (CL, red curves) and QM (blue curves) pictures, where in the former method we adopt the local-RPA graphene conductivity in eqs 1 (zero temperature) and 4 (finite temperature) for improved accuracy. For the largest island under consideration (left panel upper row), with a lateral dimension of $\sim 7.1 \mathrm{~nm}$, we obtain excellent agreement in the plasmon resonance shift and peak absorption change predicted by the CL and QM models, albeit with slight discrepancies in the spectral 
peak positions. These discrepancies become much more dramatic in smaller islands (center and right panels, upper row), with suppression of absorption emphasized in the CL model and peak energy shifts in the QM picture. To gain insight for the latter, we analyze in the lower row of Figure 2 the electronic energy-level structure obtained in the QM model upon diagonalization of the tight-binding atomistic Hamiltonian for each nanohexagon and plot the electronic distribution function before and after single-plasmon absorption. Clearly, the re-distribution of electron population among the discrete states manifests in huge shifts and the introduction of new modes in the absorption spectrum upon single-plasmon heating, consistent with spectral changes induced by the addition or removal of individual electrons in small graphene nanoislands. ${ }^{38,40}$

Ultimately, optimal single-plasmon thermo-optical switching is achieved in smaller graphene nanoislands doped with fewer electrons. Indeed, for nanohexagons containing $\sim 1000$ or fewer carbon atoms, the Fermi energy does not well describe the initial charging state in the presence of large energy gaps between discrete electronic states (see lower row in Figure 2). Instead, we consider in Figure 3 situations in which an integral number of electrons $N_{\mathrm{e}}$ are added to a graphene nanohexagon, down to doping with only a few additional electrons, and entering the so-called molecular plasmonics regime. ${ }^{37,40}$ The absorption spectra shown in the upper row of Figure 3 are dominated by quantum and finite-size effects, including peaks associated with the HOMO-LUMO transition appearing in the spectra even for undoped $\left(N_{\mathrm{e}}=0\right)$ structures. The excitation and decay of a single plasmon quantum at the frequency of this high-energy peak for various charging states results in even higher obtained electronic temperatures (see lower row of Figure 3), which manifest prominently in the shift of the lower-energy plasmonic peak appearing for $N_{\mathrm{e}}>0$, which dominates the response when $N_{\mathrm{e}} \gg 1$.

A grand goal of nonlinear optics is the realization of an all-optical switch to produce light modulations in which the modulated light is phase-locked with the light gate signal. Our proposed switch does not preserve gate-signal phase coherence, but in exchange, it pro- 

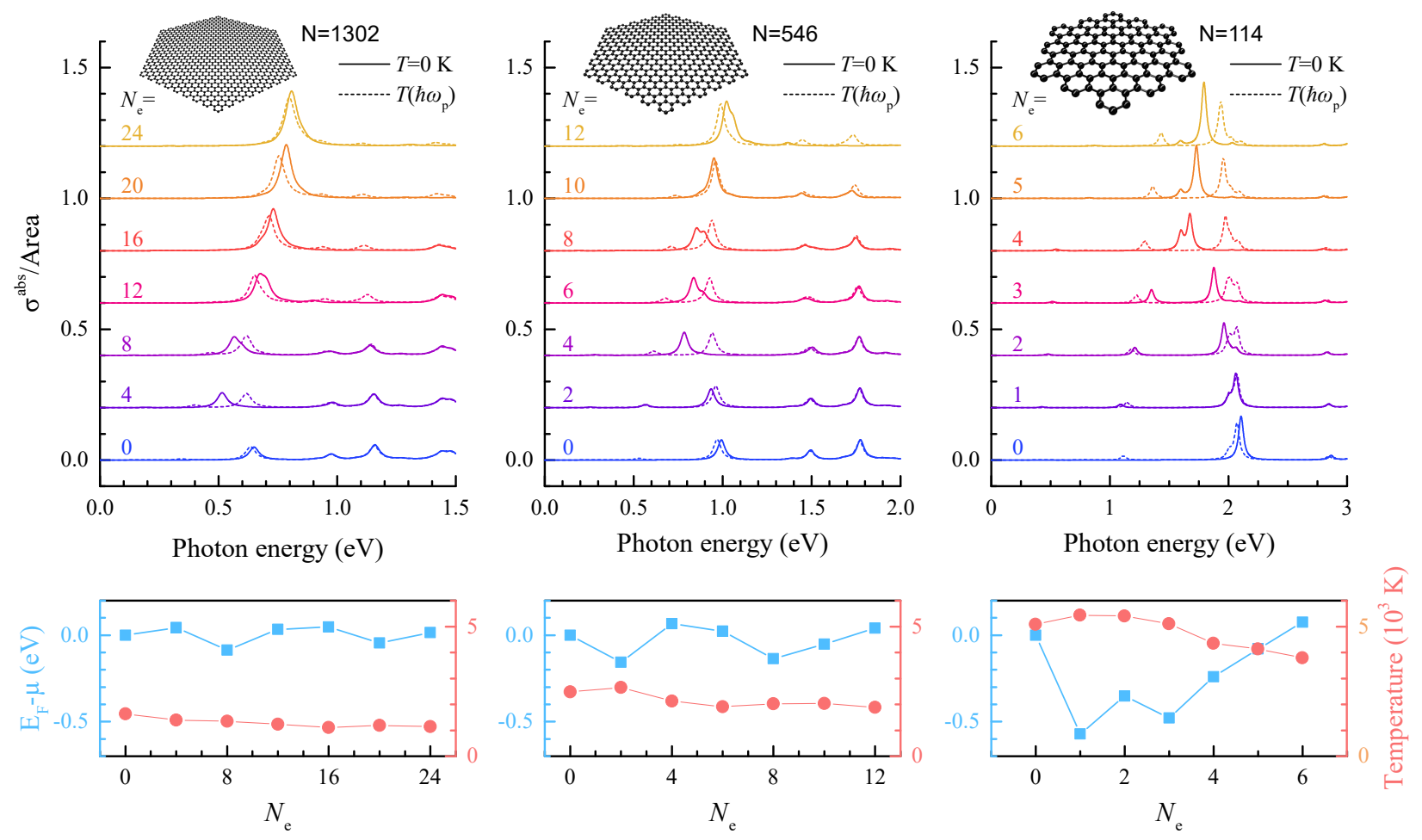

Figure 3: Thermo-optical switching by molecular plasmons. We simulate the absorption spectra of the graphene nanohexagons considered in Figure 2 doped with integral numbers of electrons $N_{\mathrm{e}}$ (upper row) before (solid curves) and after (dashed curves) the absorption of a single plasmon, with the charging states $N_{\mathrm{e}}$ indicated by the color-coordinated curves in each panel. These results are obtained in the QM description. For each hexagon we study the chemical potential shift $E_{\mathrm{F}}-\mu$ (blue squares) and electronic temperature obtained by absorbing the energy $Q=\hbar \omega_{\mathrm{p}}$ associated with the dominant absorption peak in the $T=0$ case for each charging configuration.

duces order-unity modulations with a single photon in a robust, integrable, solid-state platform: nanostructured graphene. The single-plasmon thermo-optical switching mechanism in nanostructured graphene is made possible by the intrinsically-low heat capacity of the 2D material and strong light-matter coupling of its electrically-tunable plasmon resonances, the latter enabling significant absorption even in very small structures containing only a few doping electrons. These properties are essential to achieve significant modifications in the optical response, which result from the distribution of a finite plasmon energy among a small number of electrons to produce dramatic changes in the electronic temperature and the chemical potential. Here we have considered an optimum scenario in which all of the single 
plasmon energy is distributed among thermalized electrons; in practice, a portion of this energy could be lost through inelastic scattering or coupling to phonons, thus reducing the magnitude of the effect if the graphene quality is not high. The change in optical response associated with single-plasmon absorption consists in a plasmon-blockade attained without intense external excitation, which in combination with proximal dipole emitters could open new avenues in optical sensing and quantum nano-optics.

\section{Acknowledgments}

This work has been supported in part by the Spanish MINECO (MAT2017-88492-R and SEV2015- 0522), the European Research Council (Advanced Grant 789104-eNANO), the European Commission (Graphene Flagship 696656), the Catalan CERCA Program, and Fundació Privada Cellex.

\section{References}

(1) Schuller, J. A.; Barnard, E. S.; Cai, W.; Jun, Y. C.; White, J. S.; Brongersma, M. L. Nat. Mater. 2010, 9, 193-204.

(2) Kauranen, M.; Zayats, A. V. Nat. Photon. 2012, 6, 737-748.

(3) Boyd, R. W. Nonlinear optics, 3rd ed.; Academic Press: Amsterdam, 2008.

(4) Khurgin, J. B.; Sun, G. Opt. Express 2013, 21, 27460-27480.

(5) Chang, D. E.; Vuletić, V.; Lukin, M. D. Nat. Photon. 2014, 8, 685-694.

(6) Manjavacas, A.; Liu, J. G.; Kulkarni, V.; Nordlander, P. ACS Nano 2014, 8, 7630-7638.

(7) Brongersma, M. L.; Halas, N. J.; Nordlander, P. Nat. Nanotech. 2015, 10, 25-34. 
(8) Saavedra, J. R. M.; Asenjo-Garcia, A.; García de Abajo, F. J. ACS Photon. 2016, 3, $1637-1646$.

(9) Brown, A. M.; Sundararaman, R.; Narang, P.; GoddardIII, W. A.; Atwater, H. A. ACS Nano 2016, 10, 957-966.

(10) Hartland, G. V.; Besteiro, L. V.; Johns, P.; Govorov, A. O. ACS Energy Lett. 2017, 2, 1641-1653.

(11) Baffou, G.; Quidant, R. Chem. Soc. Rev. 2014, 3898-3907.

(12) Clavero, C. Nat. Photon. 2014, 8, 95-103.

(13) Perner, M.; Bost, P.; Lemmer, U.; von Plessen, G.; Feldmann, J.; Becker, U.; Mennig, M.; Schmitt, M.; Schmidt, H. Phys. Rev. Lett. 1997, 78, 2192-2195.

(14) Voisin, C.; Del Fatti, N.; Christofilos, D.; Vallée, F. J. Phys. Chem. B 2001, 105, $2264-2280$.

(15) Khurgin, J. B.; Sun, G.; Chen, W. T.; Tsai, W.-Y.; Tsai, D. P. Sci. Rep. 2015, 5, 17899.

(16) Jablan, M.; Buljan, H.; Soljačić, M. Phys. Rev. B 2009, 80, 245435.

(17) Koppens, F. H. L.; Chang, D. E.; García de Abajo, F. J. Nano Lett. 2011, 11, 33703377.

(18) Ju, L.; Geng, B.; Horng, J.; Girit, C.; Martin, M.; Hao, Z.; Bechtel, H. A.; Liang, X.; Zettl, A.; Shen, Y. R.; Wang, F. Nat. Nanotech. 2011, 6, 630-634.

(19) Fei, Z.; Rodin, A. S.; Andreev, G. O.; Bao, W.; McLeod, A. S.; Wagner, M.; Zhang, L. M.; Zhao, Z.; Thiemens, M.; Dominguez, G.; Fogler, M. M.; Neto, A. H. C.; Lau, C. N.; Keilmann, F.; Basov, D. N. Nature 2012, 487, 82-85.

(20) Grigorenko, A. N.; Polini, M.; Novoselov, K. S. Nat. Photon. 2012, 6, 749-758. 
(21) García de Abajo, F. J. ACS Photon. 2014, 1, 135-152.

(22) Gonçalves, P. A. D.; Peres, N. M. R. An Introduction to Graphene Plasmonics; World Scientific: Singapore, 2016.

(23) Ni, G. X.; McLeod, A. S.; Sun, Z.; Wang, L.; Xiong, L.; Post, K. W.; Sunku, S. S.; Jiang, B.-Y.; Hone, J.; Dean, C. R.; Fogler, M. M.; Basov, D. N. Nature 2018, 557, $530-533$.

(24) Vafek, O. Phys. Rev. Lett. 2006, 97, 266406.

(25) Ni, G. X.; Wang, L.; Goldflam, M. D.; Wagner, M.; Fei, Z.; McLeod, A. S.; Liu, M. K.; Keilmann, F.; Özyilmaz, B.; Neto, A. H. C.; Hone, J.; Fogler, M. M.; Basov, D. N. Nat. Photon. 2016, 10, 244-248.

(26) Tan, S.; Argondizzo, A.; Wang, C.; Cui, X.; Petek, H. Phys. Rev. X 2017, 7, 011004.

(27) Yu, R.; Manjavacas, A.; García de Abajo, F. J. Nat. Commun. 2017, 8, 2.

(28) Cox, J. D.; García de Abajo, F. J. Optica 2018, 5, 429-433.

(29) Woessner, A.; Lundeberg, M. B.; Gao, Y.; Principi, A.; Alonso-González, P.; Carrega, M.; Watanabe, K.; Taniguchi, T.; Vignale, G.; Polini, M.; Hone, J.; Hillenbrand, R.; Koppens, F. H. Nat. Mater. 2015, 14, 421-425.

(30) Shi, S.-F.; Tang, T.-T.; Zeng, B.; Ju, L.; Zhou, Q.; Zettl, A.; Wang, F. Nano Lett. 2014, 14, 1578-1582.

(31) Jadidi, M. M.; König-Otto, J. C.; Winnerl, S.; Sushkov, A. B.; Drew, H. D.; Murphy, T. E.; Mittendorff, M. Nano Lett. 2016, 16, 2734-2738.

(32) Yu, R.; Cox, J. D.; Saavedra, J. R. M.; García de Abajo, F. J. ACS Photon. 2017, 4, 3106-3114. 
(33) Thongrattanasiri, S.; Manjavacas, A.; García de Abajo, F. J. ACS Nano 2012, 6, $1766-1775$.

(34) Wunsch, B.; Stauber, T.; Sols, F.; Guinea, F. New J. Phys. 2006, 8, 318.

(35) Hwang, E. H.; Das Sarma, S. Phys. Rev. B 2007, 75, 205418.

(36) Gusynin, V. P.; Sharapov, S. G.; Carbotte, J. P. Phys. Rev. Lett. 2006, 96, 256802.

(37) Manjavacas, A.; Marchesin, F.; Thongrattanasiri, S.; Koval, P.; Nordlander, P.; Sánchez-Portal, D.; García de Abajo, F. J. ACS Nano 2013, 7, 3635-3643.

(38) Manjavacas, A.; Thongrattanasiri, S.; García de Abajo, F. J. Nanophotonics 2013, 2, $139-151$.

(39) Christensen, T.; Wang, W.; Jauho, A.-P.; Wubs, M.; Mortensen, N. A. Phys. Rev. B 2014, 90, 241414(R).

(40) Lauchner, A.; Schlather, A.; Manjavacas, A.; Cui, Y.; McClain, M. J.; Stec, G. J.; García de Abajo, F. J.; Nordlander, P.; Halas, N. J. Nano Lett. 2015, 15, 6208-6214. 